\title{
Expression profile of matrix metalloproteinase-2 and -9 and their endogenous tissue inhibitors in osteonecrotic femoral heads
}

\author{
SUSANNE GRÄSSEL ${ }^{1,2}$, JOHANNES BECKMANN ${ }^{1}$, BJÖRN RATH ${ }^{1}$, \\ MANDY VOGEL ${ }^{1,2}$, JOACHIM GRIFKA ${ }^{1}$ and MARKUS TINGART ${ }^{1}$ \\ ${ }^{1}$ Department of Orthopaedic Surgery, University of Regensburg; \\ ${ }^{2}$ Centre for Medical Biotechnology, BioPark I, Regensburg, Germany
}

Received November 9, 2009; Accepted January 7, 2010

DOI: 10.3892/ijmm_00000444

\begin{abstract}
The pathogenetic mechanisms of osteonecrosis of the femoral head are unresolved to date. This study analyzed the matrix metalloprotease (MMP) and tissue inhibitor of matrix metalloprotease (TIMP) expression and activity which might add to the impaired bone matrix repair capacity affecting the balance between bone resorption and de novo bone formation in osteonecrosis of the femoral head (ONFH). Cancellous bone biopsies were taken at the femoral head and neck of patients with advanced ONFH and patients with primary osteoarthritis (OA) who were undergoing total hip arthroplasty. We assessed the gene expression levels of MMP-2 and -9 , TIMP-1 and -2 , IL-1ß, IL-6 and TNF- $\alpha$ in both biopsies. These data were corroborated by MMP activity screening, collagen profiling and ELISAs for determination of MMP, TIMP, IL-1ß, IL-6 and TNF- $\alpha$ concentrations. Gene expression rates of MMP-2 and TIMP-1 were higher in ONFH biopsies whereas TIMP-2 and MMP-9 gene expression was not significantly altered. MMP protein profile shifted towards increased biosynthesis of both, active and pro-MMP-2 in ONFH bone lysates and decreased pro-MMP-9 production. Expression profiles of pro-inflammatory cytokines and collagens in OA and ONFH bone lysates were highly similar. Increased biosynthesis and activation of MMP-2 in ONFH samples may add to shifting the bone matrix turnover balance towards resorption of bone macromolecules thereby counteracting new bone matrix production. OA- and ONFH-affected bone exhibits a similar pro-inflammatory cytokine and collagen expression profile suggesting a relationship on the molecular
\end{abstract}

Correspondence to: Dr Susanne Grässel, Department of Orthopaedic Surgery, University of Regensburg, ZMB/BioPark 1, Josef-Engert-Str. 9, D-93053 Regensburg, Germany

E-mail: susanne.graessel@klinik.uni-regensburg.de

Abbreviations: ONFH, osteonecrosis of the femoral head; OA, osteoarthritis; MMP, matrix metalloproteases; TIMP, tissue inhibitor of matrix metalloproteases; ELISA, enzyme linked immunosorbent assay; IL, interleukin; TNF- $\alpha$, tumor necrosis factor $\alpha$; PA, polyacrylamide

Key words: osteonecrosis, osteoarthritis, matrix metalloproteinase, tissue inhibitor matrix metalloprotease, collagen level of inflammation and collagenous matrix composition between both diseases.

\section{Introduction}

Osteonecrosis of the femoral head (ONFH) is a painful and progressive disorder of the hip (1). Most patients are in their middle age with a mean patient age of 38 years. For these individuals $\mathrm{ONFH}$ has a major impact on their individual lifestyle, since they often need to alter their work and leisure activities. Furthermore, progressive ONFH leads, if untreated, to a collapse of the femoral head and secondary osteoarthritis (OA) requiring total hip replacement $(2,3)$. However, a major complication of hip arthroplasty in patients with osteonecrosis is the early migration and loosening of the femoral stem. One cause discussed for this high rate of failure is a lower quality of cancellous bone in the whole proximal femur in patients with osteonecrosis $(4,5)$.

Repair capacity and bone remodelling play an important role in the progression and severity of ONFH, however, little is known about the underlying regulatory and repair mechanisms $(6,7)$. Bone remodelling is a physiological process comprised of two balanced phases, the resorption of pre-existing bone tissue by osteoclasts, followed by de novo bone formation by osteoblasts (8). The gelatinases A (matrix metalloprotease-2; MMP-2) and B (MMP-9) are described as key MMPs in bone remodelling and exhibit a broad substrate specifity towards denatured collagens and other extracellular matrix (ECM) macromolecules, thereby contributing to the degradation of collagen fibrils, basement membranes, and other suprastructures of the ECM $(9,10)$. Both gelatinases are synthesised by osteoblasts, while MMP-9 is additionally produced by osteoclasts. Expression of both MMPs in osteoblasts may be induced by pro-inflammatory cytokines such as IL-1, IL-6 and TNF- $\alpha$ whereas in osteoclasts MMP-9 expression is stimulated by RANKL (11-13). MMP-2 is known to be increased in osteoarthritic cartilage (14) while MMP-9 regulates apoptosis of hypertrophic chondrocytes and is a key regulator of growth plate angiogenesis, thus rendering MMP-9 essential for normal bone development and remodelling (15).

The major endogenous peptide inhibitors of all MMPs are tissue inhibitors of matrix metalloprotease (TIMP)- 1 to -4 . TIMP-1 and -2 are synthesised by osteoblasts and osteocytes and expression of TIMP-1 is induced by a variety of physio- 
Table I. Primer sequences for quantitative RT-PCR.

\begin{tabular}{lclll}
\hline Primer & Amplicon size (bp) & Sequence-forward & Sequence-reverse & Gene ID \\
\hline MMP-2 & 110 & gcc aat gga gac tgt ctc aag a & ttc taa ggc agc cag cag tga a & NM_004530 \\
MMP-9 & 119 & gta cca cgg cca act acg ac & gcc ttg gaa gat gaa tgg aa & NM_004994 \\
TIMP-1 & 108 & gtg tct gcg gat act tcc aca g & agc taa gct cag gct gtt cca g & NM_003254 \\
TIMP-2 & 149 & cat gat ccc gtg cta cat ctc c & atg tcg aga aac tcc tgc ttg g & NM_003255 \\
IL-6 & 113 & caa tga gga gac ttg cct gg & gca cag ctc tgg ctt gtt cc & NM_000600 \\
TNF- $\alpha$ & 123 & tcc ttc aga cac cet caa cc & cac att cct gaa tcc cag gt & NM_000594 \\
IL-1B & 149 & tca gca cct ctc aag cag aa & ccc tag gga ttg agt cca ca & NM_000576 \\
B-actin & 119 & gct aag tcc tgc cct cat ttc & ctg tgt tgg cgt aca ggt ctt & NM_001101 \\
\hline
\end{tabular}

logical stimuli such as IL-1 and TNF- $\alpha(16,17)$ among others, while TIMP-2 is not regulated by most bone remodelling agents (18). TIMP-1 is also produced by osteoclasts and its expression there is inhibited by RANKL (19). TIMP-2 is essential in MT1-MMP mediated physiological activation of MMP-2 which is regulated by a cell surface interaction of these MMPs (20). Pro-inflammatory cytokines, such as IL-6 are generally known to reduce the expression of TIMPs, while concurrently causing an increase in the expression and secretion of MMPs, cyclooxygenases and NO $(21,22)$. Additionally, IL- $1 \beta$ and TNF- $\alpha$ play important roles in bone remodelling and homeostasis. Through a variety of mechanisms these cytokines regulate osteoclast activity and osteoclastogenesis $(23,24)$.

We hypothesise that MMP-2 and -9 synthesis and activity is different in the head and neck regions of patients with ONFH vs OA and will add to the impaired bone matrix repair capacity affecting the balance between bone resorption and de novo bone formation in osteonecrosis. The aim of this study was to determine expression and activity of MMP-2, -9, TIMP-1 and -2 in the head and neck region of ONFH and OA bone samples.

\section{Materials and methods}

Patients. This study was approved by the ethics committee and informed consent was obtained from all patients included in this study. The study and control groups consist of 10 patients each. Due to the limited sample size, different patients had to be taken for quantitative RT-PCR and zymography, ELISA and SDS-PAGE in each group.

In the study group, all patients had advanced ONFH classified as stage III-IV according to the classification of Ficat (25). The mean age of the patients taken for gene expression analysis by quantitative PCR was 46 years (range, 37-56 years). Patients taken for protein analysis had a mean age of 51 years (range, 42-63 years).

The control group consisted of 10 patients with primary OA. Patients for gene expression analysis by quantitative PCR had a mean age of 65 years (range, 41-74 years), those for protein analysis had an average age also of 65 years (range, 39-86 years). There were no differences in gender between the two groups.

Bone sampling. All patients received a cementless total hip replacement using a lateral approach. Following a standardized protocol by Calder et al and Kim et al $(1,4)$, defined samples of cancellous bone $\left(1 \mathrm{~cm}^{3}\right)$ were taken from the centre of the femoral head and the base of the femoral neck (26). We chose femoral head and neck biopsies because these regions are primarily affected by osteonecrosis. Since we cannot exclude a difference in protein and gene expression profiles between these two femoral areas we prepared and analyzed them separately. Samples were either frozen at $-20^{\circ} \mathrm{C}$ for protein isolation or shock frozen in liquid nitrogen and stored at $-80^{\circ} \mathrm{C}$ for RNA isolation.

RNA extraction. From $500 \mu \mathrm{g}$ to $1.5 \mathrm{mg}$ of bone tissue pieces were rotated in a 6-ml lysis buffer for $1 \mathrm{~h}$ at room temperature. Subsequently, the lysis buffer was homogenized with a $20 \mathrm{G}$ syringe and RNA was extracted according to manufacturer's protocol (RNAaqueous ${ }^{\mathrm{TM}}$-Midi, Ambion, Germany). Crude RNA was subjected to DNase digestion as described earlier (26).

Cloning of PCR products for standard curves. Using the primers listed in Table I, 5-10 $\mu 1$ of cDNA was subjected to conventional PCR and products were ligated into PCR II Blunt-TOPO-vector (Zero Blunt ${ }^{\circledR}$ TOPO PCR Cloning kit, Invitrogen, Germany) as described (26). Identity of inserts was verified by restriction digest and sequencing. For preparation of standard curves for quantitative RT-PCR plasmids were linearized with NotI and dilution stocks of $10^{-8}-10^{-10} \mathrm{~g} / \mu 1$ were stored at $-80^{\circ} \mathrm{C}$ in single use aliquots.

Real-time quantitative $R T-P C R$. Absolute and relative quantitative RT-PCR was carried out in triplicates with $50 \mathrm{ng}$ cDNA (RNA equivalent) each with SYBR Green Dye I master mix using an ABI 7000 Prism Sequence detection system (Applied Biosystems, USA). For absolute quantification (MMP-2, TIMP-1, -2, IL-6, TNF- $\alpha$ and IL-1ß), a plasmid standard curve $\left(1 \times 10^{2}-3 \times 10^{6}\right.$ copies of the target gene) was included on each PCR plate. Contrary to relative quantification it is not necessary to normalize the genes of interest to internal control genes $(27,28)$. Relative quantification (RQ) values (MMP-9) were calculated by using ABI Prism 7000 SDS software v1.1 employing the $\Delta \Delta \mathrm{Ct}$ method with $\beta$-actin as endogenous control and OA samples as calibrator. Mean RQ values were plotted on a semi-logarithmic graph. RNA quality was checked on the Agilent Bioanalyzer 2100 (Stratagene, USA) for integrity. Primers (Table I) were designed with 
A

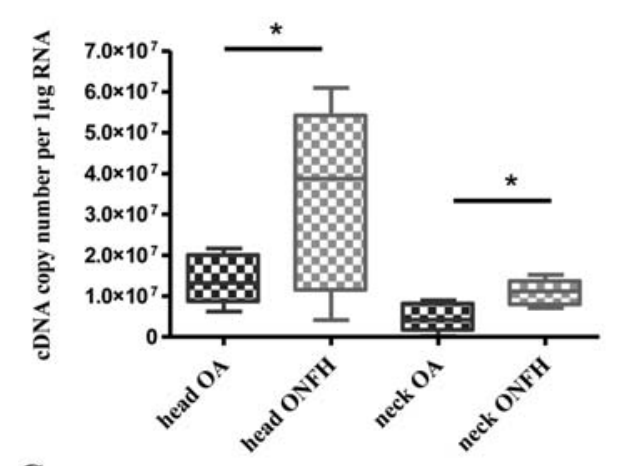

C

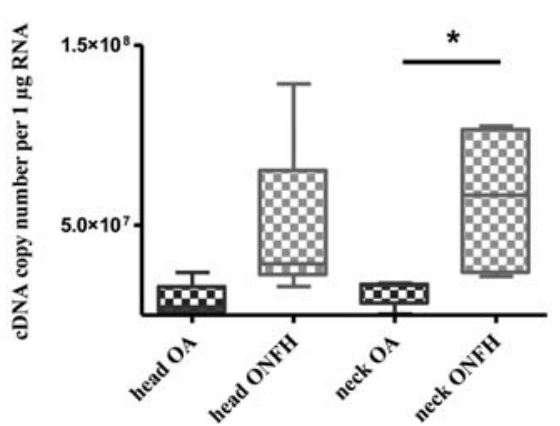

B
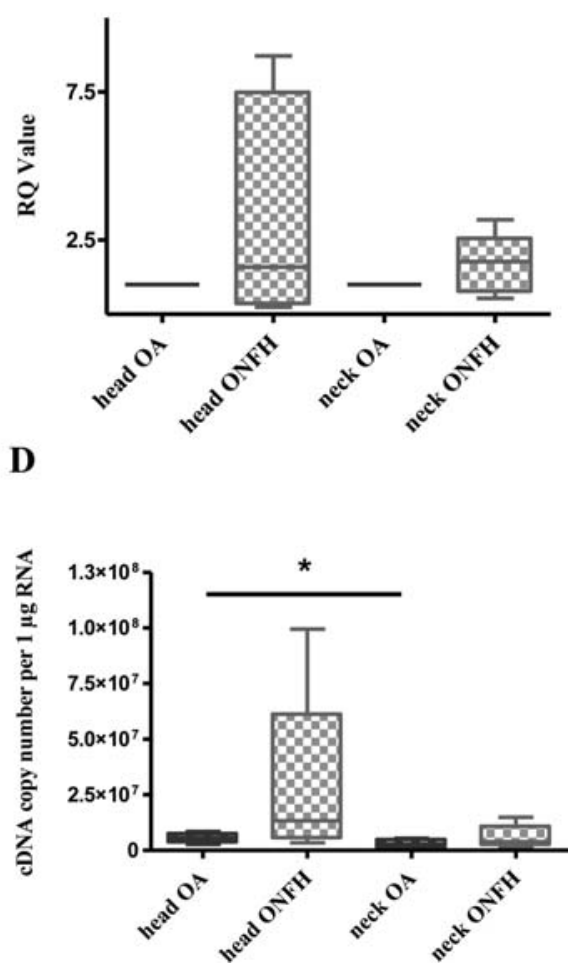

Figure 1. Gene expression level of MMP-2, -9 and TIMP-1, -2. Quantitative real-time RT-PCR, using the standard curve method, was applied for determining cDNA copy number of MMP-2 (A), TIMP-1 (C), TIMP-2 (D). cDNA copies of each gene in head and neck regions of ONFH biopsies were assessed and compared with OA samples serving as controls. Copy number was calculated in relation to $1 \mu \mathrm{g}$ RA. Relative gene expression was assessed for MMP-9 (B) using OA samples as calibrator and $\beta$-actin as endogenous control according to the $\Delta \Delta C$ method. $n=5 ;{ }^{*} \mathrm{p}<0.01$.

either Primer3 or 'Primer Express' software (Applied Biosystems, USA). All primers were manufactured at MWGBiotech, Germany.

ELISAs for collagen I, TIMP-1, -2, MMP-2, -9, IL-1 $\beta, I L-6$ and TNF- $\alpha$. TNF- $\alpha$, IL-6, TIMP-1, -2, MMP-2 and -9 protein concentrations were analyzed in $1 \mathrm{mg}$ ground bone tissue solubilized in $100 \mu 1$ lysis buffer by sandwich ELISAassay using human IL-1ß, TNF- $\alpha$, MMP-9 and -2 (R\&D Systems, USA), a human IL-6 kit (Abcam, UK), and a Collagen I kit (Chondrex, USA) according to manufacturer's instructions. Antibodies to MMP-2 and -9 detect both the active and the pro-form. Optical density after color development of respective substrates was measured at 450 or $490 \mathrm{~nm}$ with a Genios microplate reader (Tecan, Switzerland). Protein concentration was expressed in relation to $1 \mathrm{mg}$ ground bone tissue.

Collagen isolation from bone tissue. Pulverized bone (10 mg) was resuspended in $1.5 \mathrm{ml}$ pepsin solution $(2.5 \mathrm{mg} / \mathrm{ml} ; 2.5$ $3.5 \mathrm{U} / \mathrm{mg}$; Sigma Aldrich, Germany) and rotated at $4^{\circ} \mathrm{C}$ for $72 \mathrm{~h}$. After neutralizing samples with $1 \mathrm{M}$ Tris $\mathrm{HCl}$ collagens were extracted by rotation o.n. at $4^{\circ} \mathrm{C}$ with $4.5 \mathrm{M} \mathrm{NaCl}$. Collagens were precipitated at $14,000 \mathrm{rpm}$ in an Eppendorf centrifuge for $30 \mathrm{~min}$ at $4^{\circ} \mathrm{C}$ and resuspended in $400 \mu 10.4 \mathrm{M}$ $\mathrm{NaCl}$ in $0.1 \mathrm{M}$ Tris- $\mathrm{HCl}$. After adding $3 \mathrm{x}$ volume of $\mathrm{EtOH}$ absolute samples were incubated at $-20^{\circ} \mathrm{C}$ for $4 \mathrm{~h}$ and centrifuged at 14,000 rpm in an Eppendorf centrifuge for $3 \mathrm{~min}$. After air drying pellets were resuspended in $2 \mathrm{x}$ gel loading sample buffer and separated on a $4.5-15 \%$ gradient SDS-PA gel. Collagens were visualized with Coomassie Brilliant Blue R250 (Serva, Germany) staining for $1 \mathrm{~h}$. Purified bovine collagen I was a gracious gift from P. Bruckner (University of Münster, Germany).

Zymography. Aliquots of bone lysates ( $70 \mu \mathrm{g}$ total protein per sample) were mixed with equal volumes of 6 -fold concentrated sample loading buffer and subjected to electrophoresis under non-denaturing conditions on a $1 \%$ gelatin-containing 4.5$15 \%$ gradient SDS-PA gel. Gels were processed as described previously (29). For positive controls, highly purified native MMP-9 from human neutrophils (Calbiochem, Germany) and highly purified native MMP-2 from human rheumatoid synovial fibroblasts (both Calbiochem, Germany) were applied to the same gels.

Statistical analysis. The Kolmogorov-Smirnov test was used to evaluate if gene expression and ELISA parameters followed a normal (Gaussian-shaped) distribution and Levene's test was used to assess the homogeneity of variance (constant variance).

Expression of genes and ELISA parameters were compared between the two groups (osteonecrosis and osteoarthritis) using one-repeated measure ANOVA with the assumption of homogeneity of variance used as appropriate. Only where a statistical significance was found, pair-wise comparisons of the groups were performed using the t-test. A p-value $<0.05$ was considered statistically significant. 
A
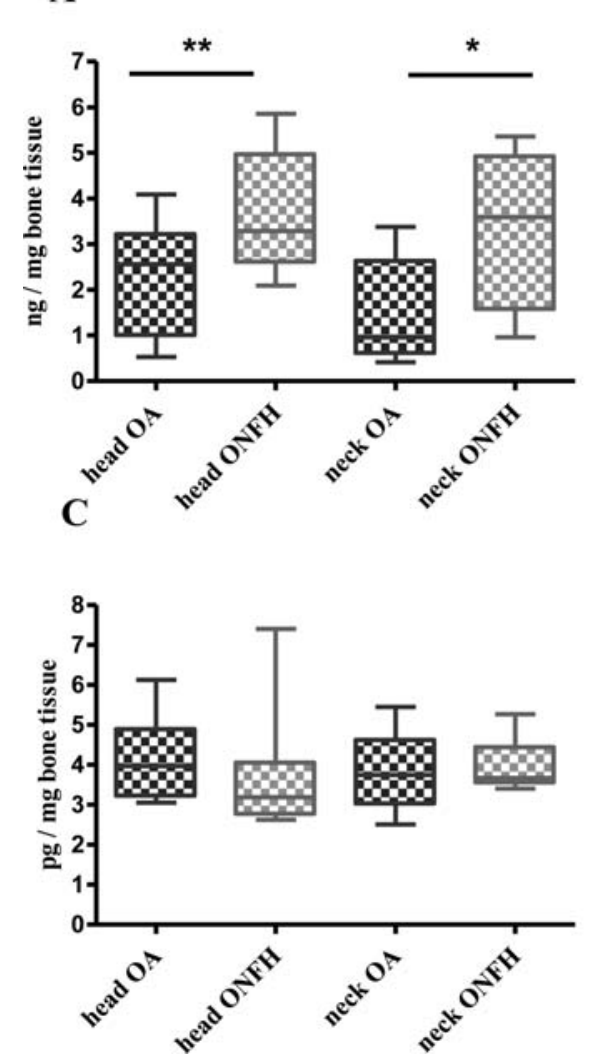

B
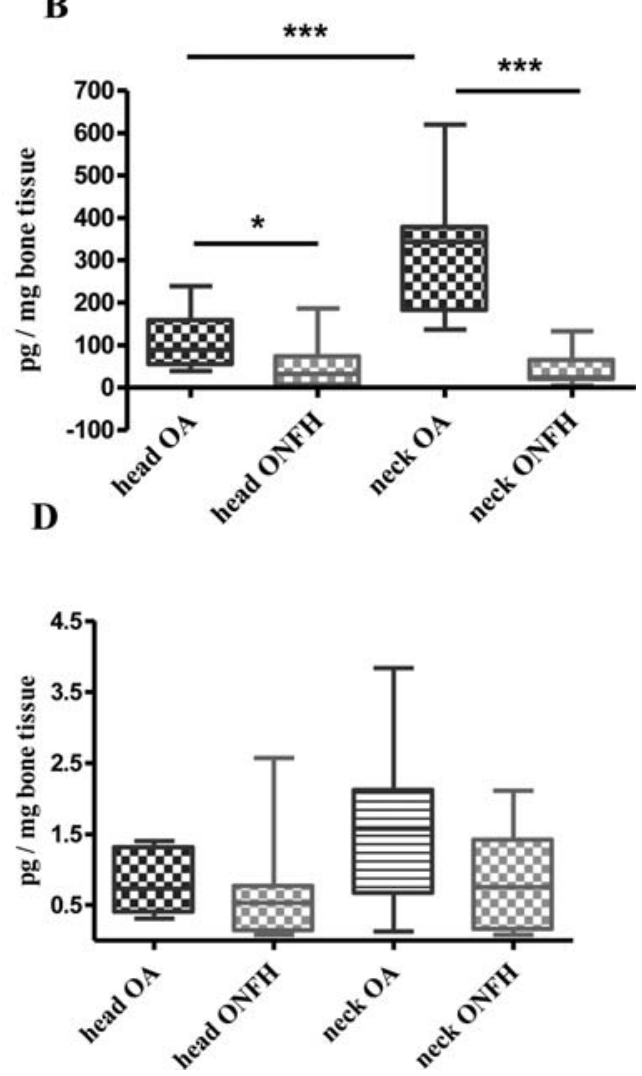

Figure 2 ELISA analysis for MMP-2, -9 and TIMP-1, -2. Ground bone tissue (1 mg) was solubilized in protein lysis buffer and applied to ELISA analysis. Antibodies to MMP-2 and -9 recognize both, the pro- and active enzymes. Protein concentrations were plotted in ng/mg tissue for MMP-2 (A), in pg/mg tissue for MMP-9 (B), TIMP-1 (C) and TIMP-2 (D). $\mathrm{n}=9 ;{ }^{*} \mathrm{p}<0.05 ;{ }^{* *} \mathrm{p}<0.01 ;{ }^{* * *} \mathrm{p}<0.001$.

Analysis of the data was performed using GraphPad Prism Version 4 (GraphPad Software, Inc., San Diego, CA, 2003) Continuous data are presented as mean \pm SD.

\section{Results}

Gene expression profile of MMP-2, -9 and TIMP-1, -2 . MMP-9 and TIMP-2 gene expression is not statistically different between OA and ONFH, while MMP-2 and TIMP-1 mRNA levels were clearly upregulated in ONFH samples (Fig. 1A-D). MMP-2 mRNA levels were profoundly elevated in both the head and neck regions, while TIMP-1 mRNA level was induced in the neck region only of patients with ONFH compared to those with OA (Fig. 1A and C). For TIMP-2 the head region in OA showed an elevated gene expression rate compared to the neck area in OA bone while for MMP-2 and TIMP-1 the mRNA levels were not statistically different between regions of the same disease (Fig. 1A, C and D). Expression rates for all 4 genes were not significantly different between the head and neck regions of the ONFH samples.

Protein expression profile of MMP-2, -9 and TIMP-1, -2. In order to analyse protein concentration, bone lysates were subjected to ELISA and data were related to $1 \mathrm{mg}$ dry weight of ground tissue. Antibodies to MMPs recognize both, proand active forms of MMP-2 and -9 . Concurrent to gene expression level, MMP-2 protein expression was induced in ONFH head and neck bone (Fig. 2A) while MMP-9 protein
A

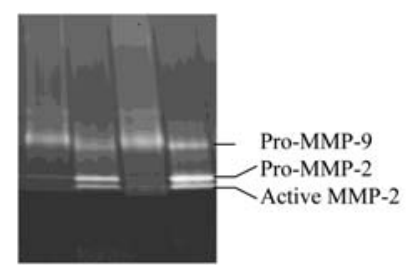

B

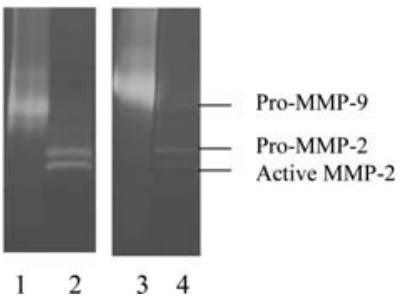

Figure 3. Gelatine zymography of bone lysates. For analysis of gelatinase activity, $70 \mu \mathrm{g}$ of head (A) and neck (B) bone lysates per lane were separated on a $15 \%$ SDS-PA gel containing $1 \%$ gelatine as substrate. A representative gel image is shown here. Lanes 1 and 3 contain OA control samples, lanes 2 and 4 ONFH samples. MMPs detected with this assay were pro-MMP-9, -2 and active MMP-2 identified according to molecular weight and purified MMP-2 and -9 used as standards. $\mathrm{n}=5$.

expression was altered inversely with an increase in OA samples. Both, head and neck from OA bone lysates contained a higher MMP-9 protein concentration as the corresponding ONFH samples. Additionally, OA neck samples contained more MMP-9 as OA head samples while these regions did not differ in MMP content in the ONFH sample (Fig. 2B). Protein expression of TIMP-1 and -2 remained unaltered 
A

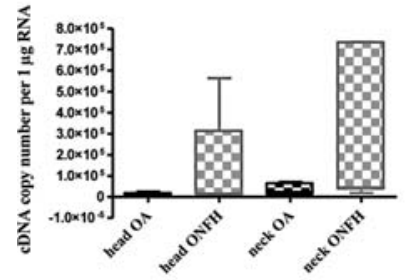

D

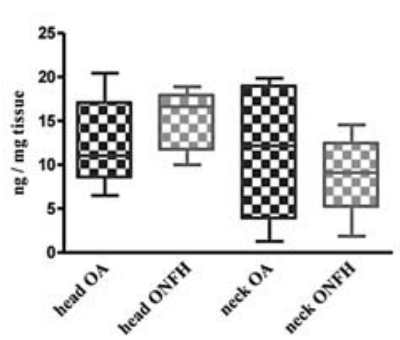

B

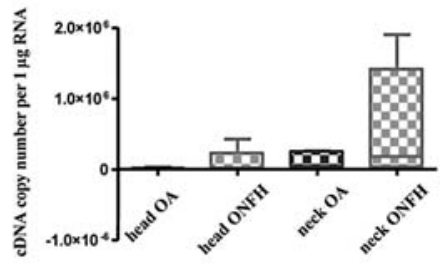

$\mathbf{E}$

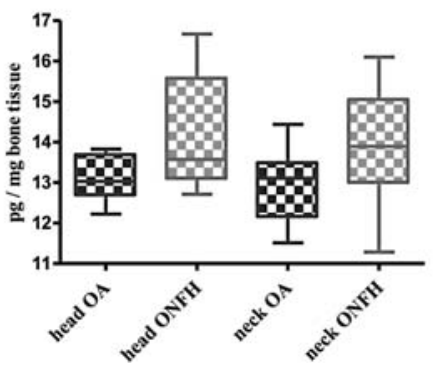

C

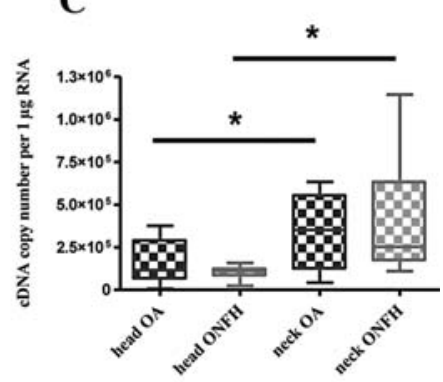

$\mathbf{F}$

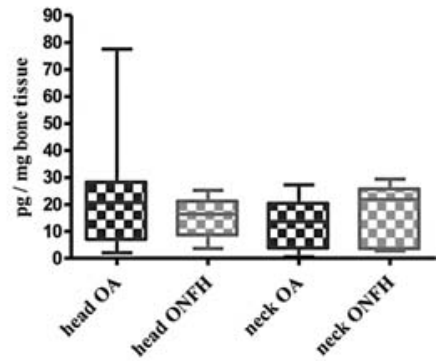

Figure 4. Gene expression level and ELISA analysis of IL-6, TNF- $\alpha$ and IL-1ß. Quantitative real-time RT-PCR, using the standard curve method, was applied for determining cDNA copy number of IL-6 (A), TNF- $\alpha$ (B) and IL-1ß (C). cDNA copies of each gene in head and neck regions of ONFH biopsies were assessed and compared with OA samples. Copy number was calculated in relation to $1 \mu \mathrm{g} \mathrm{RNA}$. $\mathrm{n}=5$. Ground bone tissue (1 mg) was solubilized in protein lysis buffer and applied to ELISA analysis. Protein concentrations were plotted in ng/mg tissue for IL-6 (D), in pg/mg tissue for TNF- $\alpha$ (E) and IL-1ß (F). $\mathrm{n}=6-10 ;{ }^{*} \mathrm{p}<0.01$.

with no differences according to region and pathology (Fig. 2C and D). Protein expression of MMPs and TIMPs was not significantly different between the head and neck regions of the ONFH samples. Notably, protein concentrations of MMPs were in the range of 50-500 pg for MMP-9 and 1-5 ng for MMP-2 while concentrations of TIMPs ranged between 0.5-5 pg per mg bone tissue lysate.

MMP-2 and -9 activation profile. Gelatine zymography confirmed differential MMP-9 and -2 biosynthesis in ONFH vs OA. Additionally, we have detected a different activation profile in bone lysates from head (Fig. 3A) and neck (Fig. 3B) when comparing ONFH and OA samples. In ONFH head and neck bone lysates both forms, pro- and active MMP-2, were detectable in increased amounts compared to corresponding OA bone samples concurrent to results of ELISA. MMP-9 remained inactive, only the pro-form was detected in all samples. As confirmed by ELISA data, bone lysates from head and neck OA samples produced more enzymes than the corresponding ONFH samples. TIMP-1 and -2 protein levels were below detection limit of reverse zymography (data not shown).

Gene and protein expression profile of $I L-1 \beta, I L-6$ and $T N F-\alpha$. Gene expression of IL-1ß, IL- 6 and TNF- $\alpha$ was neither in the head region nor in the neck area of ONFH samples significantly different from OA samples (Fig. 4A-C). IL-1ß mRNA level was higher in the neck of both, OA and ONFH samples compared to head lysates (Fig. 4C). ELISA analysis revealed for none of the cytokines a significant alteration in protein concentration (Fig. 4D-F). IL-6 protein concentration range was at the ng level, while TNF- $\alpha$ and IL- $1 \beta$ concentration ranged at the pg level.

Collagen profile. ELISA data revealed no difference in collagen I concentration per mg bone tissue between ONFH and OA samples and between head and neck (Fig. 5A) with an average concentration of collagen I at $\sim 0.5 \mu \mathrm{g}$. SDSPAGE analysis of pepsin-digested collagens revealed the presence of both $\alpha$ chains ( $\alpha 1$ and $\alpha 2)$ of collagen I in all samples analysed (Fig. 5B). No additional major fibrillar collagens were detected after Coomassie Blue staining. This collagen profile was identical for head and neck samples and did not reveal alterations in ONFH vs OA bone pepsin digests.

\section{Discussion}

In patients with $\mathrm{ONFH}$, reduced repair capacity and altered bone remodelling play an important role in progression and severity of the disease, however, little is known about the underlying molecular pathophysiology and its effect on bone metabolism (6).

The aim of our study was to determine the expression and activity profile of bone matrix degrading enzymes, MMP-2 and -9 , and their endogenous inhibitors which are known to participate in physiological and pathological bone remodelling and might affect bone matrix. Notably, MMP-2 and -9 biosynthesis is regulated in an opposite manner. While MMP-2 mRNA and protein level are clearly upregulated in ONFH samples, MMP-9 protein concentration is profoundly higher in OA bone. Xue et al described a substantially higher production of MMP-9 in peripheral monocytes and synovial 
$\mathbf{A}$

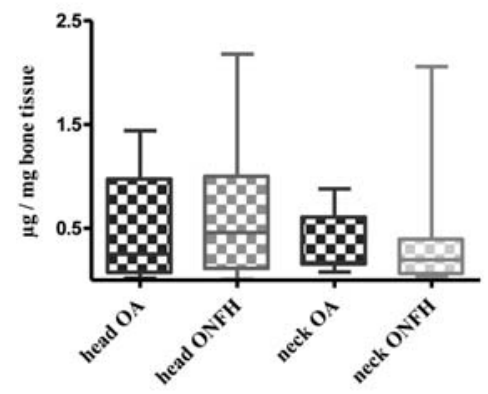

B

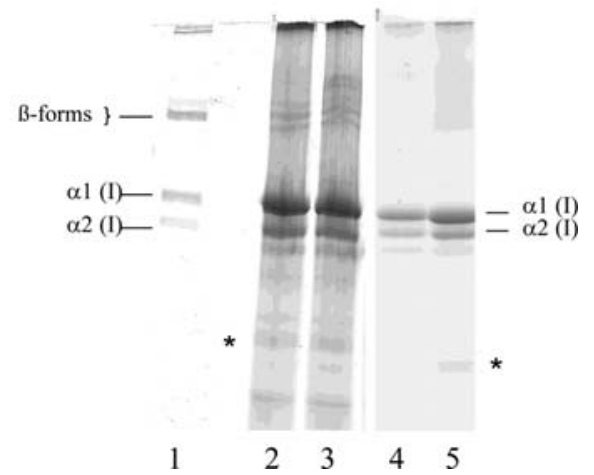

Figure 5. Collagen I profile analyzed by ELISA and SDS-PAGE. Ground bone tissue (1 mg) was subjected to pepsin and elastase digestion and assayed by a collagen I ELISA detection kit. Protein concentration was plotted in $\mu \mathrm{g} / \mathrm{mg}$ tissue $(\mathrm{A}) . \mathrm{n}=6-10$. Collagens, isolated from $10 \mathrm{mg}$ pulverized bone by pepsin digest and $\mathrm{NaCl}$ precipitation, were separated on a 4.5-15\% SDS-PA gel and stained with Coomassie brilliant blue (B). A representative gel is shown here. Lane 1 contains $5 \mu \mathrm{g}$ purified bovine collagen I ( $\alpha 1$ and $\alpha 2$ chains plus $\beta$-forms); lanes 2 and 4 contain collagens prepared from OA bone lysates and lanes 3 and 5 contain collagens prepared from ONFH bone lysates. Stars indicate pepsin bands. $\mathrm{n}=5$.

fibroblasts in rheumatoid arthritis compared to primary OA while MMP-2 production was not altered. They observed that addition of activated protein C (APC), a natural anticoagulant, inhibits the expression and production of MMP-9, but stimulates the expression and activation of MMP-2 (30). Whether APC mediated regulation of MMP-9 plays a role in $\mathrm{OA}$ or ONFH affected bones remains to be investigated, however, it is tempting to speculate that ONFH bone contains an increased number of monocytes and leukocytes which express the APC receptor (EPCR) among others (31). This might explain why we did not observe a substantial amount of active MMP-9.

The discrepancy between the MMP-9 gene expression level which remains unchanged and protein level which is higher in OA as compared to ONFH might be either explained by post-transcriptional regulation mechanisms as described for MMP-9 in HT1080 cells, a human fibrosarcoma cell line $(32,33)$ or by increased release from local neutrophils which are able to store MMP-9 in specific granules in high amounts $(34,35)$.

Our data suggest that at a late disease stage both ONFH and primary OA reveal a comparable inflammation status as the expression profile of the major pro-inflammatory cytokines IL-1ß, IL- 6 and TNF- $\alpha$ is similar. We therefore assume that alteration of MMP expression must be either promoted by additional factors or must be due to the different susceptibility of the tissues to the cytokine action because of differences in cytokine receptor expression or availability of cytokine inhibitors.

Contrary to MMP-2 and -9, TIMP-1 and -2 protein amounts in $\mathrm{ONFH}$ and OA bone biopsies were markedly lower. Apart from inhibiting MMPs, TIMPs have additional functions which include regulation of cell growth and apoptosis (36). In bone cells, TIMP-1 and -2 at low doses (10-50 ng/ml) can directly stimulate bone resorption activity independent of their ability to inhibit MMPs. They stimulate bone-resorbing activity of osteoclasts by activation of the MAPK pathway at low concentrations, however at higher doses they inhibit bone resorption likely by inhibiting responsible MMPs such as MMP-2 and -9 (37). Possibly, in primary OA and ONFH affected bone, TIMPs rather promote than inhibit bone resorption since we have detected these proteins only at very low doses (1-4 pg/mg bone tissue).

Collagen I constitutes the major collagen fraction in bone matrix and accounts for $90 \%$ of the dry mass in bone (38). Notably, we did not observe an altered collagen I content, the major fibril forming collagen in the bone matrix and the collagen protein profile including degradation products when comparing $\mathrm{ONFH}$ and $\mathrm{OA}$ bone samples. However, this observation might be due to the time point of analysis. Our samples are received at the time of total joint replacement, meaning highly advanced pathological stages in both diseases. We cannot exclude possible differences in collagen I production or degradation at earlier stages which are attenuated during progression of the diseases. Along this line, $\mu \mathrm{CT}$ analysis of structural bone parameters fail to reveal differences between OA and ONFH affected head and neck bone biopsies at this late stage of the diseases $(26,39)$.

A limitation of our study is caused by the control group, which consists of patients with primary OA who have a higher mean age than those patients with ONFH included in the study group. The ideal control group would consist of individuals with a mean age comparable to that of the patients with ONFH ( $<50$ years). Furthermore, it would have been desirable to include individuals in the control group without any bone/cartilage diseases (e.g. primary OA) or traumata of the hip joint. However, due to methodological and strict ethical restrictions we were unable to obtain bone samples of the proximal femur from healthy individuals. Therefore, comparable to the study of Calder et al (1), we decided to include individuals with primary OA who underwent total hip arthroplasty as the control group. Clearly, we have to be aware that the molecular mechanisms and expression levels of bone markers might be different in the proximal femur of individuals without any pathological changes of the hip joint.

In conclusion, we report differential MMP-2 and -9 expression in $\mathrm{ONFH}$ compared to primary OA bone biopsies. TIMP-1 and -2 protein levels are similarly low in both pathologies pointing towards stimulation of bone resorbing 
activity rather than primarily acting as endogenous inhibitors of MMP activation. We were unable to detect regional differences in protein and gene expression of gelatinases and TIMPs in corresponding ONFH head and neck regions. However, in OA affected bone protein biosynthesis of MMP-9 is increased in the neck region. OA and ONFH affected bone exhibit a similar pro-inflammatory cytokine and collagen protein profile which suggests a basic relationship on the molecular level of inflammation and collagenous matrix composition between both pathologies in advanced disease stages.

\section{Acknowledgements}

We appreciate the financial support of the Deutsche Forschungsgemeinschaft (DFG) for this study assigned to M.T. and S.G. (Az: TI 305/2-1). We thank Claudia Göttl and Anja Pasoldt for their excellent technical support.

\section{Reference}

1. Calder JD, Pearse MF and Revell PA: The extent of osteocyte death in the proximal femur of patients with osteonecrosis of the femoral head. J Bone Joint Surg Br 83: 419-422, 2001.

2. Mont MA and Hungerford DS: Non-traumatic avascular necrosis of the femoral head. J Bone Joint Surg Am 77: 459-474, 1995.

3. Tingart M, Bathis H, Perlick L, Lerch K, Luring C and Grifka J: [Therapy of femoral head osteonecrosis: results of a national survey]. Z Orthop Ihre Grenzgeb 142: 553-558, 2004.

4. Kim YH and Kim JS: Histologic analysis of acetabular and proximal femoral bone in patients with osteonecrosis of the femoral head. J Bone Joint Surg Am 86-A: 2471-2474, 2004.

5. Laroche M, Costa L, Bernard J, et al: Dual-energy X-ray absorptiometry in osteonecrosis of the femoral head. Rev Rhum Engl Ed 65: 393-396, 1998.

6. Radke S, Battmann A, Jatzke S, Eulert J, Jakob F and Schutze N: Expression of the angiomatrix and angiogenic proteins CYR61, CTGF, and VEGF in osteonecrosis of the femoral head. $J$ Orthop Res 24: 945-952, 2006.

7. Jingushi S, Lohmander LS, Shinmei M, et al: Markers of joint tissue turnover in joint fluids from hips with osteonecrosis of the femoral head. J Orthop Res 18: 728-733, 2000.

8. Karsenty G: How many factors are required to remodel bone? Nat Med 6: 970-971, 2000.

9. Murphy G, Knauper V, Atkinson S, et al: Matrix metalloproteinases in arthritic disease. Arthritis Res 4 (suppl 3): 39-49, 2002.

10. Sternlicht MD and Werb Z: How matrix metalloproteinases regulate cell behavior. Annu Rev Cell Dev Biol 17: 463-516, 2001

11. Kusano K, Miyaura C, Inada M, et al: Regulation of matrix metalloproteinases (MMP-2, $-3,-9$, and -13) by interleukin-1 and interleukin-6 in mouse calvaria: association of MMP induction with bone resorption. Endocrinology 139: 1338-1345, 1998.

12. Lorenzo JA, Pilbeam CC, Kalinowski JF and Hibbs MS: Production of both $92-$ and $72-\mathrm{kDa}$ gelatinases by bone cells. Matrix 12: 282-290, 1992

13. Wittrant Y, Theoleyre S, Couillaud S, Dunstan C, Heymann D and Redini F: Regulation of osteoclast protease expression by RANKL. Biochem Biophys Res Commun 310: 774-778, 2003.

14. Duerr S, Stremme S, Soeder S, Bau B and Aigner T: MMP-2/ gelatinase $\mathrm{A}$ is a gene product of human adult articular chondrocytes and is increased in osteoarthritic cartilage. Clin Exp Rheumatol 22: 603-608, 2004.

15. Vu TH, Shipley JM, Bergers G, et al: MMP-9/gelatinase B is a key regulator of growth plate angiogenesis and apoptosis of hypertrophic chondrocytes. Cell 93: 411-422, 1998.

16. Panagakos FS and Kumar S: Differentiation of human osteoblastic cells in culture: modulation of proteases by extracellular matrix and tumor necrosis factor-alpha. Inflammation 19: 423-443, 1995.

17. De Bart AC, Quax PH, Lowik CW and Verheijen JH: Regulation of plasminogen activation, matrix metalloproteinases and urokinase-type plasminogen activator-mediated extracellular matrix degradation in human osteosarcoma cell line MG63 by interleukin-1 alpha. J Bone Miner Res 10: 1374-1384, 1995.
18. Cook TF, Burke JS, Bergman KD, Quinn CO, Jeffrey JJ and Partridge NC: Cloning and regulation of rat tissue inhibitor of metalloproteinases-2 in osteoblastic cells. Arch Biochem Biophys 311: 313-320, 1994.

19. Bord S, Horner A, Beeton CA, Hembry RM and Compston JE: Tissue inhibitor of matrix metalloproteinase-1 (TIMP-1) distribution in normal and pathological human bone. Bone 24: 229-235, 1999.

20. Butler GS, Butler MJ, Atkinson SJ, et al: The TIMP2 membrane type 1 metalloproteinase 'receptor' regulates the concentration and efficient activation of progelatinase A. A kinetic study. J Biol Chem 273: 871-880, 1998.

21. Goldring MB: The role of cytokines as inflammatory mediators in osteoarthritis: lessons from animal models. Connect Tissue Res 40: 1-11, 1999.

22. De Ceuninck F, Dassencourt L and Anract P: The inflammatory side of human chondrocytes unveiled by antibody microarrays. Biochem Biophys Res Commun 323: 960-969, 2004.

23. Stashenko P, Dewhirst FE, Peros WJ, Kent RL and Ago JM: Synergistic interactions between interleukin 1, tumor necrosis factor, and lymphotoxin in bone resorption. J Immunol 138: 1464-1468, 1987.

24. Bertolini DR, Nedwin GE, Bringman TS, Smith DD and Mundy GR: Stimulation of bone resorption and inhibition of bone formation in vitro by human tumour necrosis factors. Nature 319 : 516-518, 1986.

25. Ficat RP: Idiopathic bone necrosis of the femoral head. Early diagnosis and treatment. J Bone Joint Surg Br 67: 3-9, 1985.

26. Tingart M, Beckmann J, Opolka A, et al: Influence of factors regulating bone formation and remodeling on bone quality in osteonecrosis of the femoral head. Calcif Tissue Int 82: 300-308, 2008.

27. Wong ML and Medrano JF: Real-time PCR for mRNA quantitation. Biotechniques 39: 75-85, 2005.

28. Bustin SA: Absolute quantification of mRNA using real-time reverse transcription polymerase chain reaction assays. J Mol Endocrinol 25: 169-193, 2000.

29. Dreier R, Wallace S, Fuchs S, Bruckner P and Grassel S: Paracrine interactions of chondrocytes and macrophages in cartilage degradation: articular chondrocytes provide factors that activate macrophage-derived pro-gelatinase B (pro-MMP-9). J Cell Sci 114: 3813-3822, 2001.

30. Xue M, March L, Sambrook PN and Jackson CJ: Differential regulation of matrix metalloproteinase 2 and matrix metalloproteinase 9 by activated protein C: relevance to inflammation in rheumatoid arthritis. Arthritis Rheum 56: 2864-2874, 2007.

31. Galligan L, Livingstone W, Volkov Y, et al: Characterization of protein $\mathrm{C}$ receptor expression in monocytes. Br J Haematol 115: 408-414, 2001.

32. Fahling M, Steege A, Perlewitz A, et al: Role of nucleolin in posttranscriptional control of MMP-9 expression. Biochim Biophys Acta 1731: 32-40, 2005.

33. Limaye AM, Desai KV, Chavalmane AK and Kondaiah P: Regulation of mRNAs encoding MMP-9 and MMP-2, and their inhibitors TIMP- 1 and TIMP-2 by androgens in the rat ventral prostate. Mol Cell Endocrinol 294: 10-18, 2008.

34. Xu M, Scott JE, Liu KZ, et al: The influence of nicotine on granulocytic differentiation - inhibition of the oxidative burst and bacterial killing and increased matrix metalloproteinase- 9 release. BMC Cell Biol 9: 19-25, 2008.

35. Pruijt JF, Fibbe WE, Laterveer L. et al: Prevention of interleukin-8induced mobilization of hematopoietic progenitor cells in rhesus monkeys by inhibitory antibodies against the metalloproteinase gelatinase B (MMP-9). Proc Natl Acad Sci USA 96: 10863-10868, 1999.

36. Baker AH, Edwards DR and Murphy G: Metalloproteinase inhibitors: biological actions and therapeutic opportunities. J Cell Sci 115: 3719-3727, 2002.

37. Sobue T, Hakeda Y, Kobayashi Y, et al: Tissue inhibitor of metalloproteinases 1 and 2 directly stimulate the bone-resorbing activity of isolated mature osteoclasts. J Bone Miner Res 16: 2205-2214, 2001.

38. Kivirikko KI: Collagen biosynthesis: a mini-review cluster. Matrix Biol 16: 355-356, 1998.

39. Beckmann J, Matsuura M, Grassel S, Kock F, Grifka J and Tingart M: A muCT analysis of the femoral bone stock in osteonecrosis of the femoral head compared to osteoarthrosis. Arch Orthop Trauma Surg 129: 501-505, 2009. 